EDITORIAL

\title{
A COVID-19 from Laboratory Suspicion to Confirmatory Tests: Controversial Debatable
}

\author{
Yasser Mohammed Hassanain Elsayed \\ Critical Care Unit, Fraskour Central Hospital, Egypt
}

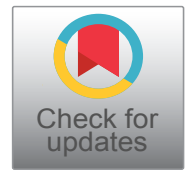

*Corresponding author: Yasser Mohammed Hassanain Elsayed, Critical Care Unit, Fraskour Central Hospital, Damietta Health Affairs, Egyptian Ministry of Health (MOH), Damietta, Egypt

\section{Background}

\section{Between laboratory suspicion and tests confirma- tion}

A coronavirus-2019 (COVID-19) patient is currently balanced between laboratory suspicion and confirmatory tests. This is still controversial debatable. Sometimes, the cost and test availability push the physician towards this area. The disease severity, high infectivity, test sensitivity, and specificity are other playing factors. The strong willingness for mortality predictors is an essential tool for clinicians to rapidly triage the severe COVID-19 patients at admission into critical care departments [1]. The COVID-19 pandemic has currently carried a significant impact on diagnostic laboratory services [2]. The contemporary literature had concentrated on checking tests that are specifically regarding the diagnosis of COVID-19 infection [2]. The tests are undoubtedly important epidemiological ways, especially, for the evaluation of the prevalence of asymptomatic patients of COVID-19 [3]. Rapid and accurate laboratory diagnosis of active COVID-19 infection is a hallmark in pandemic control [4]. The use of advised specimen types and laboratory testing techniques in the right clinical scenario could be challenging for general practitioners [4]. Utilizing molecular testing and immunoassays revealing viral antigens or antibodies is the base [2]. Synchronously, non-specific laboratory tests are the most popular demanded tests in patients with suspected COVID-19 infection to help evaluate the consequence systemic complications of COVID-19 infection [2] (Figure 1).

\section{Test accuracy towards sensitivity and specificity in COVID-19}

Accuracy is an index for assessment of both specificity and selectivity in COVID-19 infection. This is because test errors may be false positives (i.e., the test is positive, but the virus is not present) or false negatives, (the test is negative, but the virus is present) [1]. Sensitivity is a mark for test accuracy for the presence of the COVID-19 virus. This test necessitates a minimum level of viral load to cause a positive result. Indeed, a $90 \%$ sensitive test will accurately pick out $90 \%$ of COVID-19 infections, but the remaining $10 \%$ will be missed (a false negative). Even relatively high sensitivity rates can result in higher false negatives rate in inhabitants with depressed prevalence rates to COVID-19 infections [1].

Specificity is a mark for the test negativity in the COVID-19 virus. A highly specific test accurately will pick up only the COVID-19 virus in a query. Non-specific tests pick up other viruses as well. A $90 \%$ specific test will correctly recognize $90 \%$ of an uninfected population by the COVID-19 virus, but the remaining $10 \%$ will be in the area of false-positive result [5]. However, lowspecificity tests have a low positive predictive value (PPV) if prevalence is low [6]. RT-PCR is the most precise diagnostic test in COVID-19 infections [7]. COVID-19 infections ideally possess high sensitivity and specificity in a laboratory setting, but the sensitivity in a single study decreased to $66-88 \%$ clinically [8].

\section{ICU hospitalization and severity of COVID-19 in- fection using the common laboratory tests}

Hospitalization is more likely in the more severe 


\section{BOUID-19}

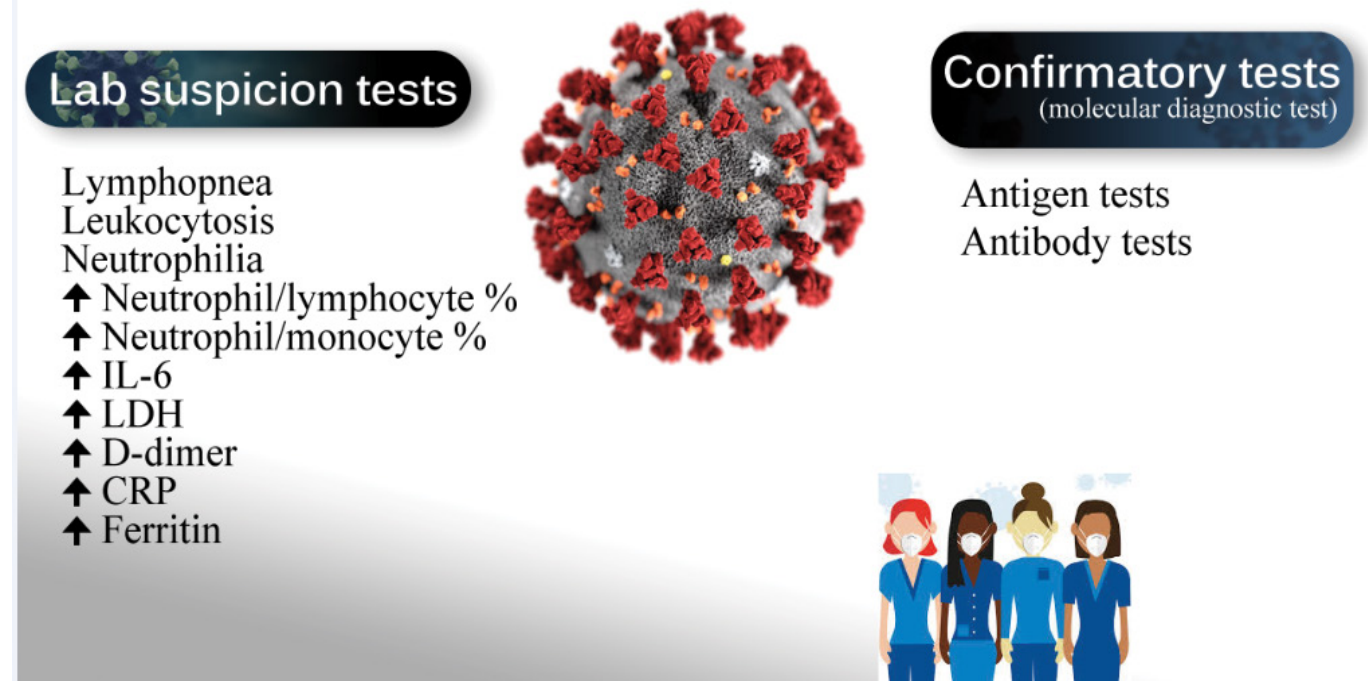

Figure 1: Showing the balance of COVID-19 patients between laboratory suspicion and confirmatory tests.

Table 1: Common clinical laboratory tests in the emergency setting of suspected COVID-19 infection.

\begin{tabular}{|l|l|}
\hline Lab tests & $\begin{array}{l}\text { Abnormality in COVID-19 } \\
\text { patients }\end{array}$ \\
\hline Albumin & Decreased \\
\hline Lactate dehydrogenase & Increased \\
\hline Alanine aminotransferase & Increased \\
\hline Aspartate aminotransferase & Increased \\
\hline Total bilirubin & Increased \\
\hline Creatinine & Increased \\
\hline C-reactive protein & Increased \\
\hline Cardiac troponin & Increased \\
\hline Urea & Predicted to be increased \\
\hline Blood gas panel & $\begin{array}{l}\text { Predicted to have increased } \\
\text { and decreased levels }\end{array}$ \\
\hline Ferritin & Increased \\
\hline Procalcitonin & Increased \\
\hline IL-6 & Increased \\
\hline
\end{tabular}

Modified from the International Federation of Clinical Chemistry and Laboratory Medicine (IFCC) list and ref [10].

cases of COVID-19. Approximately, $20 \%$ of cases of COVID-19 will be needing critical care admission [9]. The recognition and differentiation between the more severe COVID-19 versus non-severe patients using the general laboratory tests will be entirely valuable for clinicians in predicting the disease progression [9]. Meta-analyses revealed a significant fall in lymphocyte, monocyte, and eosinophil, hemoglobin, platelet, albumin, serum $\mathrm{Na}+$, lymphocyte to C-reactive protein \% (LCR), leukocyte to C-reactive protein \% (LeCR), leukocyte to IL-6 \% (LeIR), and an increase in the neutrophil, serum glutamic pyruvic transaminase (SGPT), serum glutamic oxaloacetic transaminase (SGOT), total bilirubin, blood urea nitrogen, creatinine, erythrocyte sedimentation rate (ESR), C-reactive protein (CRP), Procalcitonin (PCT), lactate dehydrogenase (LDH), fibrinogen, prothrombin time (PT), d-dimer, glucose level, and neutrophil to lymphocyte ratio (NLR) in the severe group compared with the non-severe group9. No significant changes in white blood cells $(\mathrm{WBC})$, creatine kinase (CK), troponin I, myoglobin, IL-6, and K between the two groups were noted (Table 1) [10]. This meta-analysis gives evidence for the triage of severe cases of COVID-19 relied on laboratory test results at the decision of critical care admission [9].

\section{Numeric and morphologic complete blood count differential changes in COVID-19 patients}

Interestingly, all COVID-19 patients showed remarkable numeric and morphologic white blood cell (WBC) abnormalities, which can differentiate the mild from severe disease infection [11]. So, hospitalized COVID-19 patients must subject to a daily complete blood count $(C B C)$ and manual WBC differential for observing numerical and morphologic abnormalities. These changes are predictive of poor outcome and signs of disease progression [11].

Abnormal WBC morphology is most vigorous in monocytes and lymphocytes with moremild COVID-19 disease but these abnormalities were missing with COVID-19 disease progression [11]. The research changes in values that indicative severe acute respiratory syndrome in both COVID-19-positive and COVID-19negative of ICU patients were recorded. There were significant differences in morphology-associated the two viruses, including elevated RNA material in monocytes, decreased RNA material in lymphocytes, and minimal hypogranular neutrophils [11]. In severe COVID-19 
disease, there were remarkable neutrophilia and Iymphopenia, especially, in ICU patients [11]. So, severe disease is usually accompanied by lymphopenia and leukocytosis [12]. Both abnormal cellular and humoral immunity were essential findings of non-survivors with COVID-19 patients [13]. Thus, neutrophilia and lymphocytopenia were immunity-linked risk factors guide for mortality in COVID-19 patients [13].

Current reports had revealed that the presence of leukocytosis in a proportion of COVID-19 patients presentation. Unfortunately, clinical data regards these patients are rare. An elder COVID-19 patient presented with underlying chronic disorders is more liable to have leukocytosis. These patients are more at risk to develop severe illness, higher need for ICU admission, and raised mortality rate [14].

COVID-19 patients with lymphopenia must be assessed for suitable management and the ICU admission [15]. Lymphopenia and a raised neutrophil/lymphocyte $\%$ are the most harmonious abnormal hemocytometric changes and modifications that may deteriorate in the severity of COVID-19 disease [16].

There is a strong need for recognizing the mortality predictors that permit clinicians to rapidly triage severe COVID-19 patients into ICUs at hospitalization [1]. Indeed, the lymphocyte-to-neutrophil ratio (LNR) and neutrophil-to-monocyte ratio (NMR) are good predictors for hospitalization in severe COVID-19 infection [1].

\section{Inflammatory and biological markers with COVID-19 patients}

A fourfold increase of $d$-dimer at hospitalization greater than $2.0 \mu \mathrm{g} / \mathrm{mL}$ is an effective predictor for in-hospital mortality in patients with COVID-19. It is helpful as an early biomarker that indicated to improve the management of COVID-19 patients [17]. Autopsies reported increasing in CRP level in patients who died of COVID-19 infection. So, it is a good biomarker for assessing disease lethality [18]. COVID-19 patients with elevated CRP levels and elevated LDH should be assessed for suitable management and the ICU admission [15].

There is a profuse cytokine releasing with hyperferritinemia which suggests that a COVID-19 infection is part of the hyperferritinemic syndrome series19. Indeed, marked elevated ferritin levels can present in other non- COVID-19 diseases such as hemophagocytic lymphohistiocytosis, macrophage activation syndrome, adult-onset Still's disease, catastrophic antiphospholipid syndrome, and septic shock [19]. Several studies have shown the immunomodulatory results of ferritin and its relation to mortality and continued inflammatory effects [1]. The raised concentration of free iron is a tissue hazardous which associated with redox damage and ending with fibrosis. Iron chelation is essentially considered in the management of iron overload. Iron chelation also possesses an anti-viral and anti-fibrotic activity [19]. All four types gathered under the term "hyperferritinemic syndromes" is sharing the clinical and laboratory data with the severe form of COVID-19 several features. This similar sharing between COVID-19 and "hyperferritinemic syndromes" is meaning that the severe COVID-19 is the fifth one of hyperferritinemic syndromes [20]. Decreased CD4+ T cells and Low C3 are immunological indices to assess the risk and predicting mortality of COVID-19 patients [13].

\section{COVID-19 confirmation; antibody versus antigen tests controversial}

As the antibody tests are emerged, researchers will be able to observe if persons have a history of infection with SARS-CoV-2 can be re-infected and the type of this re-infection [3]. The diagnosis of COVID-19 has essentially relied on the typical clinical presentation, radiological bilateral pneumonia, and history of exposure to the infected COVID-19 patients [21]. The clinical symptoms and signs are very similar to other viral infections. So, the differential diagnosis from other viral infections is an essential [22]. Non-quantitative antibody assay is especially useful in epidemiological surveys in which infectivity rate in a specific category of people can be determined [4].

COVID-19 virus confirmation is only by positive nucleic acid test of SARS-CoV-2 using several types of samples [21]. Nucleic acid amplification test (NAAT) is the technique of choice for a diagnosis of an active COVID-19 infection. The use of real-time RT-PCR assay to detect SARS-CoV-2 RNA from the URT is the choice initial diagnostic test [4].

The NAAT assays essentially target the SARS-CoV-2 nucleocapsid $(N)$, envelope $(E)$, and spike $(S)$ genes, and regions in the first open reading frame (orf1a and orf1b), and the RNA-dependent RNA polymerase (RdRp) gene [4].

Reverse-transcriptase-polymerase-chain-reaction (RT-PCR) is the most frequently used technique for SARS-CoV-2 diagnosis is aiming at ORF1ab, N, or E genes [21]. It is the most commonly used molecular diagnostic test for the diagnosis of COVID-19 in biological samples [23]. However, there is no generally currently accepted test is available. So, many countries adopting different test strategies [23].

Oropharyngeal and nasopharyngeal swabs are the most commonly taken specimens [21]. To estimate whether a person is recently infected with SARS-CoV-2 who may be needing the RT-PCR test [3]. The clinical diagnosis of COVID-19 mainly relies on laboratory detection of COVID-19 virus nucleic acid. However, the results are affected by virus levels and types of sample collection. But, unfortunately, false-negative 
are common results. Moreover, various studies have revealed changes in the laboratory detection data of COVID-19 patients [22].

A COVID-19 confirmation is depending on the detection of either presence of the virus or of antibodies as a response to COVID-19 infection [24,25]. Tests for viral presence are used to diagnose individual cases and to allow for public health institutes the followup and monitoring of viral outbreaks. Antibody tests instead show whether someone once had the disease. These tests are less helpful for the establishment of a diagnosis of current COVID-19 infections due to delayed development of the antibodies for a few weeks after infection [26]. It is commonly used to measure disease prevalence rather than fatality rate [27].

So, antibody tests must not be used to diagnose a current COVID-19 infection unless there was a delay in viral testing. The test may be negative in the first three weeks after infection until antibodies formation [26]. Whatever the test positive or negative for COVID-19 in either a viral or an antibody test, a patient still should take care to protect yourself and others [26].

The suggested question; How much protection (immunity) antibodies to the COVID-19 virus might provide against getting infected again? Confirmed and suspected COVID-19 cases of reinfection had been recorded [26]. In some patients, antibodies may persist in the bloodstream for many years, but others disappear away [28]. The potency and protective period of SARSCoV-2 antibodies are still not been established [27,29]. So, a positive antibody test is not meaning a good immunity to a future infection. Whatever production of enough antibodies in the mild or asymptomatic COVID-19 infections has not been established [30]. Serological tests for SARS-CoV-2 are more needed to improve the number of cases of COVID-19 rather than asymptomatic or have recovered cases [25]. A highly sensitive test will catch all true positive cases. A highly specific test will exclude all true negative cases [25].

Serologic antibody tests are used to confirm suspected cases and unknown infected cases. About 25 $\%$ of SARS-CoV-2 infection may be passing as mild or no symptoms27. Other strains of coronaviruses cannot be causing a positive viral test for SARS-CoV-2 [26].

Antibody tests also can be used to evaluate if a sector of a people has once been infected and calculate of mortality rate [27]. Dissimilarity to polymerase chain reaction (PCR) tests (or molecular or nucleic acidbased tests) in the throat or nasal swabs, antibody tests can't identify the active SARS-CoV-2 infections [27]. Alternatively, antibody tests show markers of the immune response (IgM and IgG antibodies) in blood for more than a week after start sickness [27].

In this early pandemic of COVID-19, serology tests can be used to screen donor blood antibodies to
SARS-CoV-2 [27]. Experimentally, Plasma containingantibodies in recovered cases is transfused to severely diseased patients as a treatment known as convalescent plasma [27].

The most frequent recognizable antibodies are IgM and IgG. IgM antibodies are commonly seen few days post-initial infection [31]. IgG antibodies usually appeared within 10-14 days post-infection but peaked by end of the fourth week post-infection [32,33]. Unfortunately, this drama of antibody pathway can be detectable with other non-SARS-CoV-2 infections. This is usually with IgM or sometimes IgG, or together with IgG, or not happening at all [34]. Moreover, median IgM detection exists within 5 days after presentations, but IgG has existed within a median of 14 days after presentations [35]. IgG levels significantly decline after two or three months [36].

Genetic tests stratify infection earlier than antibody tests. One-third of a positive genetic test associated with a positive antibody test on the seventh of initial infection [30]. Antigen assessment tests reveal the existence of SARS-CoV-2 viral proteins in respiratory samples. The available commercial kits mostly need nasal cavity or nasopharynx samples. But the other samples such as saliva are still under research. Unfortunately, these tests do not involve target protein amplification and are usually less sensitive than NAATs. Variant sensitivities on using these antigen tests should be taken into consideration which is varies significantly with different assays. The advantage of antigen detection tests is a simple to perform, accessible on limited NAAT tests, and more beneficial where there is an expected high viral load [4].

SARS-CoV-2 antigens can be revealed before the clinical presentation of COVID-19 and have more rapid test results. But unfortunately, it is less sensitive than PCR tests for the COVID-19 virus [37]. The average specificity of antigen tests is $99.5 \%$, and the average sensitivity is $56.8 \%$, but there is a huge variation in sensitivity tests starting from 0 to $94 \%$ and depending on various companies [38]. Depending on the WHO reports, there are cross- sensitivity similar antigen tests for a flu-like disease that ranges between 34\% and $80 \%$ [39]. So, more than $50 \%$ of infected COVID-19 patients will be missed by such tests [39]. Some scientists doubt in benefits of antigen tests for COVID-1939, but others have the support that high sensitivity of antigen tests if there are high viral load and contagious people [40]. Routine antigen tests can rapidly recognize asymptomatic contagious cases rather than followup PCR in confirmatory diagnosis [41]. Antigen tests may be one way to scale up testing too much greater levels [28]. Isothermal nucleic acid amplification tests are getting only one process for only one sample at a time per machine. RT-PCR tests are the most decisive but it is time-consuming, energy missing and need to 
Table 2: Showing variability of sensitivity depending on site of sample collection in 205 COVID-19 Wuhan patients [44].

\begin{tabular}{|l|l|}
\hline Sample's source & Sensitivity \\
\hline Bronchoalveolar lavage fluid specimens & $93 \%(14 / 15)$ \\
\hline Sputum & $72 \%(75 / 104)$ \\
\hline Nasal swabs & $63 \%(5 / 8)$ \\
\hline Fibro bronchoscope brush biopsy & $46 \%(6 / 13)$ \\
\hline Pharyngeal swabs & $32 \%(126 / 398)$ \\
\hline Feces & $29 \%(44 / 153)$ \\
\hline Blood & $1 \%(3 / 307)$ \\
\hline
\end{tabular}

train personnel for the tests [28]. "There will never be the ability on a [PCR] test to do $\mathbf{3 0 0}$ million tests a day or to test everybody before they go to work or school," Deborah Birx, head of the White House Coronavirus Task Force, said on 17 April 2020. "But there might be with the antigen test [42]." Swabs of respiratory viruses often lack enough antigen material to be detectable [43]. Sensitivity is variable depending on the location of sample collection for COVID-19 patients [44] (Table 2). Upper respiratory tract (URT) specimens are an advisable sample in clinical diagnosis of active disease in symptomatic cases in early-stage of COVID-19 infections or asymptomatic cases [4]. Lower respiratory tract (LRT) specimens have remarkable viral loads with likely to result in positive tests versus URT specimens [4]. Fecal specimens are indicated in patients with negative respiratory tract specimens even there is strong clinical suspicion [4]. Saliva is containing live COVID-19 viruses which is a pool of the lower respiratory tract. So, using saliva is a logically reasonable test [23]. Interestingly, the content of salivary COVID-19 or viral load appears to be highest in the first week post-symptom onset. This emphasizes the significance of saliva as a possible source of viral infection. It is possibly detected in the saliva during 25 days post-symptom onset. So, assessment for viral clearance and prophylactic care during handling during this period is acceptable. Hence, the clinician should understand that the anti-COVID-19 antibodies might also be present in human saliva despite this has not been recorded with no probable using for diagnostics and disease monitoring [23].

However, antigens react with the strips and produce visual readout results. The used timeless than half-hour can deliver results with care and does not need highcost equipment or e trained personnel [28]. This is good for asymptomatic patients with little nasal discharge [28,39].

Single-stranded RNA (S-S RNA) virus uses angiotensinconverting enzyme 2 (ACE2) as a receptor for invasion of the host cell. Overexpression of ACE2 is usually seen in diabetic, hypertensive, and cardiac patients that more probable to SARS-CoV-2 infection. The selection of drugs repurposing is usually based on the scientific rationale [45]. The repurposed drugs such as Chloroquine, hydroxychloroquine, tenofovir, remdesivir, ribavirin, darunavir, oseltamivir, umifenovir, favipiravir, anakinra, and baricitinib are suggested drugs in clinical trials in the treatment of COVID-19 cases. Despite these drugs are linked to a different category but already approved for a diverse indication [45].

Quidel's "Sofia 2 SARS Antigen FIA" test [46] is a lateral flow test that uses monoclonal antibodies to detect the virus's nucleocapsid (N) protein [47]. The result is depending on a device using immunofluorescence [47]. It is easier and fewer costs but inaccurate than nucleic acid tests. It can give results in 15 minutes [47]. But unfortunately, a false negative result exists if there are positive antigen test, below the antigen test detection limit, needing confirmation with a nucleic acid test [47].

\section{Conclusion and Recommendations}

A COVID-19 patient is currently balanced between laboratory suspicion and confirmatory tests. Sensitivity, specificity, and accuracy of laboratory suspicion and confirmatory tests are relatively variable. The author thinks that whatever the test positive or negative for COVID-19 in either a viral or an antibody test, the patient still should take care to protect yourself and others. The potency and protective period of SARS-CoV-2 antibodies are still not been established.

\section{Acknowledgement}

I wish to thank Dr. Ameer Mekkawy M.sc. for technical support.

\section{Conflicts of Interest}

There are no conflicts of interest.

\section{References}

1. Rizo-Téllez SA, Méndez-García LA, Flores-Rebollo $C$, Alba-Flores F, Alcántara-Suárez R, et al. (2020) The neutrophil-to-monocyte ratio and lymphocyte-to-neutrophil ratio at admission predict in-hospital mortality in mexican patients with severe SARS-CoV-2 infection (Covid-19). Microorganisms 8: 1560.

2. Borai $A(2020)$ Non-specific laboratory tests in patients with covid-19. Ann Clin Lab Sci 50: 528-535.

3. Burki TK (2020) Testing for COVID-19. Lancet Respir Med J 8: e63-e64.

4. Lai CKC, Lam W (2021) Laboratory testing for the diagnosis of COVID-19. Biochem Biophys Res Commun 538: 226230.

5. Maureen F (2020) COVID-19 tests are far from perfect, but accuracy isn't the biggest problem. Popular Science.

6. American Medical Association (2020) Serological testing for SARS-CoV-2 antibodies.

7. Chen LY (2020) Heartbreak in the streets of wuhan. Bloomberg Business Week.

8. Sturts A (2020) Comparing RT-PCR and chest CT for diagnosing COVID-19.

9. Sulmaz Ghahramani, Reza Tabrizi, Kamran B Lankarani, Seyyed Mohammad Amin Kashani, Shahla Rezaei, et al. (2020) Laboratory features of severe vs. non-severe 
COVID-19 patients in Asian populations: A systematic review and meta-analysis. Eur J Med Res 25: 30.

10. Giuseppe Lippi, Mario Plebani (2020) The critical role of laboratory medicine during coronavirus disease 2019 (COVID-19) and other viral outbreaks. Clin Chem Lab Med 58: 1063-1069.

11. Pozdnyakova O, Connell NT, Battinelli EM, Connors JM, Fell G, et al. (2021) Clinical significance of CBC and WBC morphology in the diagnosis and clinical course of COVID-19 infection. Am J Clin Pathol 155: 364-375.

12. Huang G, Kovalic AJ, Graber CJ (2020) Prognostic value of leukocytosis and lymphopenia for coronavirus disease severity. Emerg Infect Dis 26: 1839-1841.

13. Zhao Y, Nie HX, Hu K, Wu XJ, Zhang YT, et al. (2020) Abnormal immunity of non-survivors with COVID-19: Predictors for mortality. Infect Dis Poverty 9:108.

14. Zhao K, Li R, Wu X, Zhao Y, Wang T, et al. (2020) Clinical features in 52 patients with COVID-19 who have increased leukocyte count: A retrospective analysis. Eur J Clin Microbiol Infect Dis 39: 2279-2287.

15. Zhang ZL, Hou YL, Li DT, Li FZ (2020) Laboratory findings of COVID-19: A systematic review and meta-analysis. Scand J Clin Lab Invest 80: 441-447.

16. Khartabil TA, Russcher $H$, van der Ven $A$, de Rijke YB (2020) A summary of the diagnostic and prognostic value of hemocytometry markers in COVID-19 patients. Crit Rev Clin Lab Sci 57: 415-431.

17. Zhang L, Yan X, Fan Q, Liu H, Liu X, et al. (2020) D-dimer levels on admission to predict in-hospital mortality in patients with Covid-19. J Thromb Haemost 18: 1324-1329.

18. Sahu BR, Kampa RK, Padhi A, Panda AK (2020) C-reactive protein: A promising biomarker for poor prognosis in COVID-19 infection. Clin Chim Acta 509: 91-94.

19. Perricone C, Bartoloni E, Bursi R, Cafaro G, Guidelli GM, et al. (2020) COVID-19 as part of the hyperferritinemic syndromes: The role of iron depletion therapy. Immunol Res 68: 213-224.

20. Colafrancesco S, Alessandri C, Conti F, Priori R (2020) COVID-19 gone bad: A new character in the spectrum of the hyperferritinemic syndrome? Autoimmun Rev 19: 102573.

21. Wang X, Tan L, Wang X, Liu W, Lu Y, et al. (2020) Comparison of nasopharyngeal and oropharyngeal swabs for SARS-CoV-2 detection in 353 patients received tests with both specimens simultaneously. Int $\mathrm{J}$ Infect Dis 94: 107-109.

22. Yun H, Sun Z, Wu J, Tang A, Hu M, et al. (2020) Laboratory data analysis of novel coronavirus (COVID-19) screening in 2510 patients. Clin Chim Acta 507: 94-97.

23. Sapkota D, Thapa S, Hasséus B, Jensen JL (2020) Saliva testing for COVID-19? Br Dent J 228: 658-659.

24. U.S. Centers for Disease Control and Prevention (2020) Coronavirus disease 2019 (COVID-19).

25. Kobokovich A, West R, Gronvall G (2020) Global progress on COVID-19 serology-based testing. Johns Hopkins Center for Health Security.

26. U.S. Centers for Disease Control and Prevention (2020) Test for Past Infection.

27. Abbasi J (2020) The promise and peril of antibody testing for COVID-19. JAMA 323: 1881-1883.
28. Technology Networks (2020) Developing antibodies and antigens for COVID-19 diagnostics.

29. Scientific American (2020) What immunity to COVID-19 really means.

30. Deeks JJ, Dinnes J, Takwoingi Y, Davenport C, Spijker R, et al. (2020) Antibody tests for identification of current and past infection with SARS-CoV-2. The Cochrane Database Syst Rev 6: CD013652.

31. Food and Drug Administration (2020) Cellex emergency use authorization.

32. The New York Times (2020) Will an antibody test allow us to go back to school or work?

33. FDA (2020) Mount sinai emergency use authorization.

34. Bauer G (2020) The variability of the serological response to SARS-corona virus-2: Potential resolution of ambiguity through determination of avidity (functional affinity). Journal of Medical Virology 93: 311-322.

35. Ravi N, Cortade DL, Ng E, Wang SX (2020) Diagnostics for SARS-CoV-2 detection: A comprehensive review of the FDA-EUA COVID-19 testing landscape. Biosens Bioelectron 165: 112454.

36. Goudouris ES (2021) Laboratory diagnosis of COVID-19. J Pediatr 97: 7-12.

37. Guglielmi G (2020) Fast coronavirus tests: What they can and can't do. Nature 585: 496-498.

38. Dinnes J, Deeks JJ, Adriano A, Berhane S, Davenport C, et al. (2020) Rapid, point-of-care antigen and molecular-based tests for diagnosis of SARS-CoV-2 infection. Cochrane Database Syst Rev 8: CD013705.

39. CNN (2020) What to know about the three main types of coronavirus tests.

40. Jonathan S (2020) Failing the coronavirus-testing test. Harvard Magazine.

41. Food and Drug Administration (2020) coronavirus (COVID-19) update: FDA authorizes first antigen test to help in the rapid detection of the virus that causes COVID-19 in patients.

42. AAAS (2020) NIH launches competition to speed COVID-19 diagnostics.

43. Whitehouse gov (2020) Remarks by president Trump, vice president Pence, and members of the coronavirus task force in press briefing.

44. Wang W, Xu Y, Gao R, Lu R, Han K, et al. (2020) Detection of SARS-CoV-2 in different types of clinical specimens. JAMA 323: 1843-1844.

45. Kumar P, Sah AK, Tripathi G, Kashyap A, Tripathi A, et al. (2021) Role of ACE2 receptor and the landscape of treatment options from convalescent plasma therapy to the drug repurposing in COVID-19. Mol Cell Biochem 476: 553574.

46. Food and Drug Administration (2020) Roche diagnostics $\mathrm{GmbH}$ elecsys anti-SARS-CoV-2.

47. Food and Drug Administration (2020) For use under the emergency use authorization (EUA) only for in vitro diagnostic use. Sofia 2 SARS Antigen FIA. 